\title{
A Smart City Case Study: Dynamic Management of Road Lanes
}

\author{
Chen Wang, Bertrand David, and René Chalon \\ Université de Lyon, CNRS, Ecole Centrale de Lyon, LIRIS, UMR5205, \\ 36 avenue Guy de Collongue, F-69134 Ecully Cedex, France \\ \{Chen.Wang, Bertrand. David, Rene. Chalon\} @ec-lyon.fr
}

\begin{abstract}
The SMART CITY is an important field for ubiquitous computing (UC), ambient intelligence (AmI), connected vehicles (CV), and new styles of User Interfaces, mainly mobile. Data vitalization related to in-city data collection and their appropriate diffusion to city actors (private and professional) and their services (applications) is one issue. In a more precise and specific context of dynamic lane allocation system, which is presented in this paper, we describe the use of Location-Based services and Internet of Things, as well as the User Interfaces proposed. A simulation environment allows us to conduct a first validation of the system and to study acceptability of User Interfaces before in-thefield deployment.
\end{abstract}

Keywords: smart city, ambient intelligence, ubiquitous computing, data vitalization, location-based services, mobile internet, internet of things, dynamic lane allocation.

\section{Introduction - Smart City Issue}

In recent years, the concept of "smart cities" has emerged to describe how investments in human and social capital and modern Information and Communication Technologies (ICT) infrastructure and e-services fuel sustainable growth and quality of life, enabled by wise management of natural resources and via participative government.

In Smart City systems there are two extreme approaches: 1/ Opportunistic systems allowing access to collected information and its "vitalization" by integration - interaction - aggregation in a non-predetermined way; 2/ Well-defined systems able to solve identified problems. While in our international China-France academic research project we study these two approaches, in this paper we focus on explaining one typical Smart City system, the goal of which is to solve a precise problem:

- More appropriate traffic management, avoiding congestion by better allocation of traffic lanes

Before providing a detailed description of our system, the next section gives an overview of related works. We then present and discuss two main techniques used in our system: Location-Based Services (LBS) and Internet of Things (IoT). 
According to Z. Xiong [1], the "Smart City" principle in opportunistic perception is based on the concept of "Data Vitalization". The idea is to give data life, to combine separated data by avoiding information islands, to build a combination between each type of data, and to increase utilization of data. The main issue concerns the sharing and integration of data that are separated due to their type or different collection methods. Contextual access and use of these data are fundamental, as well as location-based services, the goal of which is to increase data and services utility and contextual and location-based usability.

In another extreme perception, based on precise demands to be solved, the system architecture is the same [1], but diversity and "vitalization" are not the main goals. In this case, direct usability, reliability and performance are more important. In our case, we studied a precise situation characterized by appropriate communication and collaboration between several categories of users (private and professional), their vehicles and corresponding services.

\section{ICT Techniques to Be Used: Internet of Things and Location-Based Services}

\subsection{Internet of Things}

The Internet of things allows static and dynamic environmental objects to communicate and update real situations. The basic idea behind the Internet of Things (IoT) concept is the pervasive presence around us of a variety of things or objects - such as Radio-Frequency IDentification (RFID) tags, sensors, actuators, mobile phones, etc. which, through unique addressing schemes, are able to interact with each other and cooperate with their neighbors to reach common goals [2].

At first glance, the concept of IoT recalls the idea of Ambient Intelligence (AmI) and Ubiquitous Computing (UC). The former refers to electronic environments that are sensitive and responsive to the presence of people. In an AmI world, devices work together to support people in carrying out their everyday life activities, tasks and rituals in an easy and natural way using information and intelligence that is hidden in the network connecting these devices; when the devices are smaller and more integrated into our environment, only the user interface remains perceivable by users [3]. The UC focuses on the omnipresence of processing devices, which are small, inexpensive, robust, networked, and distributed at all scales [4]. The concept of IoT is closely linked to AmI and UC, but its central issues are to make a full interoperability of interconnected devices possible, providing them with an ever higher degree of smartness by enabling their adaptation and autonomous behavior, while guaranteeing trust, privacy and security [5].

Although IoT has not been fully deployed, industrial, standardization and research bodies believe that it could have a huge impact on the behavior and social life of potential users. Possible application domains could be domestic and working fields such as domotics and healthcare, and could also include industrial manufacturing, intelligent transportation and business management. The US National Intelligence Council 
has placed the IoT in the list of six "Disruptive Civil Technologies" that will have potential impacts on US national power [6]. According to the NIC, "by 2005, Internet nodes may reside in everyday things - food packages, furniture, paper documents, and more", "popular demand combined with technology advances could drive wide-spread diffusion of an internet of things that could, like the present Internet, contribute invaluably to economic development".

\subsection{Location-Based Services}

Location-Based Services (LBS) provide personalized services to mobile users according to their locations. In actual fact, LBS has evolved from online map services and other internet Geographical Information Systems (GIS) applications to the current form where more lightweight mobile devices (smart-phones, wearable computers, etc.) are used to deliver services, thanks to the development of Global Positioning Systems (GPS) and other location sensing technologies [7]. The authors of [8] view the evolution of LBS in other perspectives, (a) from reactive to proactive, (b) from self- to cross-referencing and (c) from single- to multi-target: reactive LBS are explicitly invoked by the user, while proactive LBS are automatically initiated when a predefined event occurs; user and target coincide in self-referencing LBS, while cross-referencing LBS make use of one target location for service-provisioning of another user; the major focus is on tracking one target's position in single-target LBS, while in multi-target LBS, focus is rather on interrelating the positions of several targets. We will see, later, in the following sections, how proactive and crossreferencing LBS contribute to our system design when integrating IoT that allows static and dynamic environmental objects to communicate and collaborate with each other.

Just as in other interactive systems, user modeling is a basic consideration in LBS as the services finally delivered must correspond to user needs. We have to make sure right from the start who the users are and what kind of services they need. One description of user modeling could be "the acquisition or exploitation of explicit, consultable models of either human users of systems or the computational agents which constitute the system" [9]. We are pleased to extend these "computational agents" to the objects in the IoT, since an object could either provide or consume LBS in the form of a "Web Service".

Location modeling is also a central part of LBS. One static location can be represented either as a geometric coordinate such as " $48^{\circ} 51^{\prime} 29.6^{\prime \prime} \mathrm{N}, 2^{\circ} 17^{\prime} 40.2^{\prime \prime} \mathrm{E}$ ", in the World Geodetic System (WGS) [10], or as a symbolic expression such as "Champ de Mars, 5 Avenue Anatole France, 75007 Paris". The difference comes from the two distinctive models for representing space [7]: geometric models that "treat location and objects as points, areas and volumes within a reference coordinate system" and symbolic models that consider locations as sets and located objects as members of sets, so that "interrelationships are established among a set of locations and a set of located objects". Geometric models are largely adopted by GIS applications, while 
symbolic models are more easily accepted by the general public. Depending on the scenarios of applications or the degrees of accuracy, it would be helpful to carry out some combinations or merges of these two models. For example, a semantic location model was developed by the authors of [11], able to create location hierarchy (belonging to one location and being a boundary point from and into which an entity can leave and enter) and exit hierarchy automatically without manual intervention. Dynamic location data, in other words spatio-temporal data, could provide more information (especially in real time management systems), making modeling and processing of spatio-temporal data hot topics in LBS.

\section{Using These Techniques in the Case of Dynamic Management of Road Lanes}

Our study concerns dynamic management of road traffic, which is regularly increasing both in towns and outside agglomerations. A first approach to allow increase in traffic leads to solutions such as increasing the number of lanes, while a second aims to segment traffic according to categories (private vehicles, heavy vehicles, public transport, priority vehicles) by proposing specific development and traffic rules, with, in particular, the creation of specialized lanes for high-occupancy vehicles (bus, tram, trolley). This second choice can lead to satisfying solutions provided that there is enough space.

When space is lacking and the frequency of this type of specialized traffic is not sufficient, there is a sense of waste and poor management. A third solution is the dynamic allocation of lanes to different types of transport. A significant work of data gathering, analysis and classification was carried out by J. Nouvier of CERTU (Centre for studies on networks, transports, urbanism and public construction) [12]. He provides a large amount of varied solutions, from the more physical (ad hoc movement of low walls with trucks) to the more informational (signposts with variable displays), enabling a greater or lesser speed of dynamicity.

Today it is true that telematic or embedded and/or mobile ICT can provide solutions leading to a very high dynamicity (clear a lane for a bus or an ambulance in real time) provided that users are sufficiently informed and that regulations are complied with in terms of transport (or suggestions to modify it) and, in particular, of user safety. Hereafter we give a brief description of our ICT vision, in a system-perspective.

"Dynamic circulation lane allocation" aims at providing a system designed to share circulation lanes dynamically between public and rescue service transportation (buses, fire-fighters and ambulances) and personal vehicle transportation in order to share traffic lanes appropriately in the context of traffic jams and lack of space (impossibility or inadequacy of static allocation of circulation lanes). When there are no buses, all lanes are allocated to the general public. When a bus approaches and on the bus driver's request, the right-hand lane is reserved for it. Once the bus has passed, the reserved lane is returned to the general public (Fig. 1). 


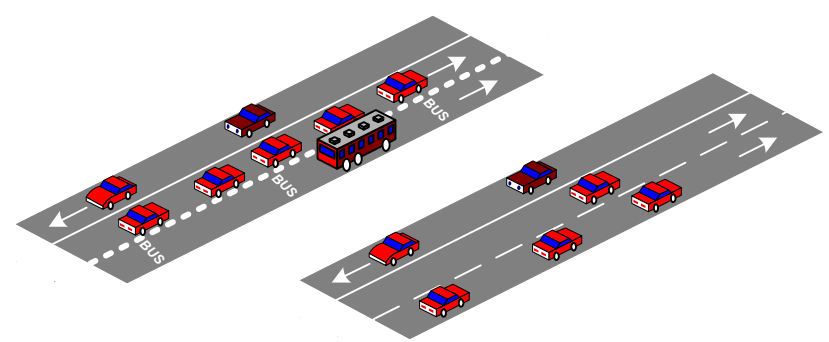

Fig. 1. Dynamic circulation lane allocation

\subsection{System Vision}

An overview of our application is shown in figure 2. Its goal is to collect inenvironment information, communicate with active and passive users (and their vehicles), and take into account authorities' decisions. In the street, sensors collecting traffic evaluation and specific demands from authorized drivers (buses, emergency vehicles, etc.) are able to communicate observed situations to the system. The system decides on the appropriate action, commands dynamically the position of vertical and horizontal signaling, and propagates the new system state to all users by appropriate media (radio, GSM, Wi-Fi, etc.) to inform them as to expected behavior (use of reserved lanes for a bus by non-priority drivers if no bus is expected, leaving a lane which is now reserved for priority drivers when a bus is approaching, etc.).

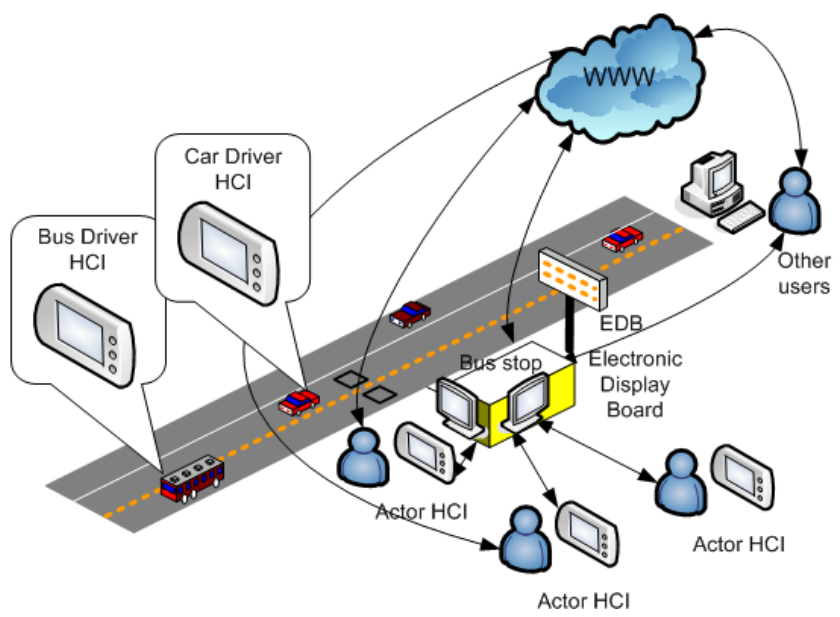

Fig. 2. Overview of the dynamic lane allocation environment 


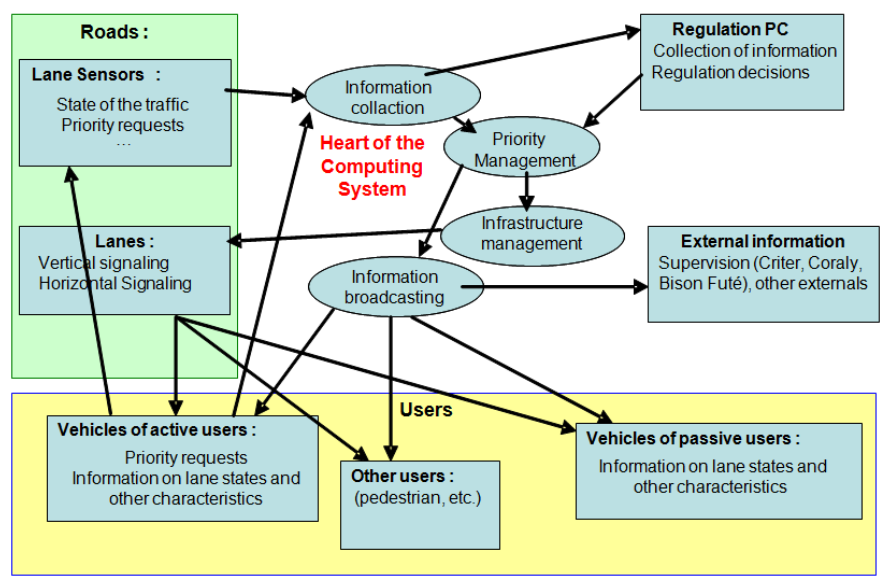

Fig. 3. Diagram of the system architecture

The corresponding dynamic management system for lanes is based on a set of sensors, displayers and activators located in strategic places: on the lane (sensors as well as displayers and activators), with the active users (requestors such as bus drivers, ambulances, firemen, possibly trucks) involved, passive users received the imposed modifications (displayers only) and external traffic regulators, as shown in figure 2. All the elements must be integrated in the global system, collecting, aggregating, processing and diffusing the appropriate information to all users. This system vision (Fig. 3) brings into play the main elements, namely:

- the sensors in the lane concerned by the collection of information regarding state of the traffic and priority requests;

- the vehicles of users who request priority and who receive information regarding the state of the lanes (allocation of lanes, authorized or not dynamic priority requests) for on-board display;

- 2 wireless networks: UMTS, WiFi, etc. EDB - Electronic Display Boards;

- the vehicles of passive users who cannot take action, but who receive information regarding the state of the lanes (whether or not they are dynamically allocated to different categories of users: prohibited lane, lane reserved for priority vehicles, unmarked lanes);

- regulation, a vital component of coordination, which chooses the mode of lane management (static allocation in the event of heavy traffic or dynamic allocation in the event of infrequent priority seeking). It does not systematically intervene in management (automatic functioning is preferred), but only intervenes to change the management mode;

- the lanes receiving information regarding the propagation of both vertical (variable message signs) and horizontal (on the road) signaling;

- the system of dynamic management of so-called priority roads; management includes the following main components: collection of information, management of priorities, management of lanes, and diffusion of information;

- the information transmission system (the information network). 


\subsection{HCI Vision}

With respect to the users identified in the system vision, it is important to deal with the user aspect as soon as possible, by designing and implementing appropriate human-computer interfaces: as observed, active users (requestors - beneficiaries of the system), passive users (subjected to the system), deciders (regulation command center) and all users via vertical and horizontal signaling must receive useful information to act appropriately. It is essential that this information and these actions comply with the work context and the corresponding requirements:

- in the vehicle, compatibility with the dual task - driving and managing; in a regulation work station for example, it is efficiency which dominates;

- when information is displayed outside, it has to be compatible with meteorological constraints of visibility - legibility.

Design of these HCIs therefore also forms an important part of the system and conditions its acceptability. Simulator-based ergonomic studies of acceptability for this kind of interface as a complementary task to driving will be presented in the next section.

General Description of the Simulator. We first simulate the system, with the appropriate simplifications that need to be chosen. This lays down the base for implementation of the digital simulator. The main elements presented in the diagram in figure 3 are included in the simulation. There are two types of view in the simulation: vertical views and horizontal views. In the vertical point of view (overlook), as in figure 4, the road is represented as a rectangular area and is made up of several lanes ( 3 for the

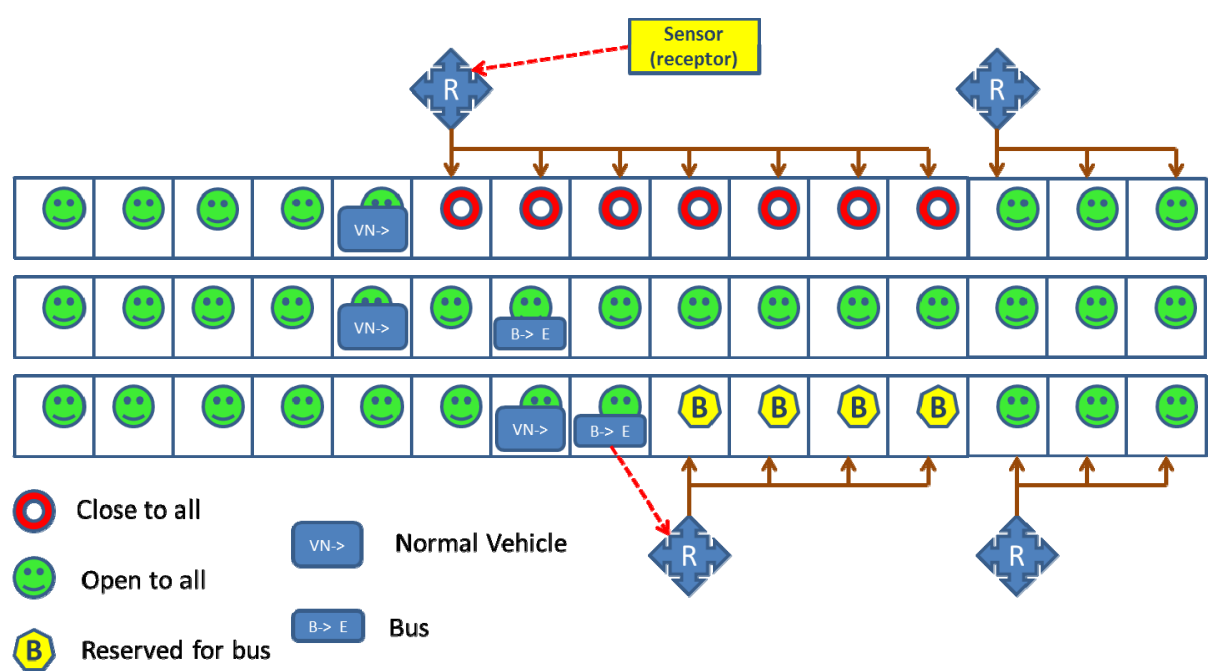

Fig. 4. Simulation scenario view and graphic editor tool 
actual version), with each lane in straight line form. Each lane is divided into a series of squares for relative location modeling, so that the positions of vehicles and signaling facilities as well as sensors could be found easily. The squares can also receive horizontal signaling to inform users about the current status of lane allocation, for example "open to all", "reserved for bus" or "closed to all", with the number of squares showing the length of the allocation area.

The horizontal view is from the interior of the vehicle, as in figure 5; a driving scene is presented in real time with the corresponding simulated elements. The driver can see the vertical signaling on the roadside as well as the horizontal signaling on the ground from the front window of the vehicle. Moreover, the surrounding circumstances are also reflected in the rearview mirrors of the vehicle. An embedded interface is designed for passive users and active users to receive information and/or request priority.

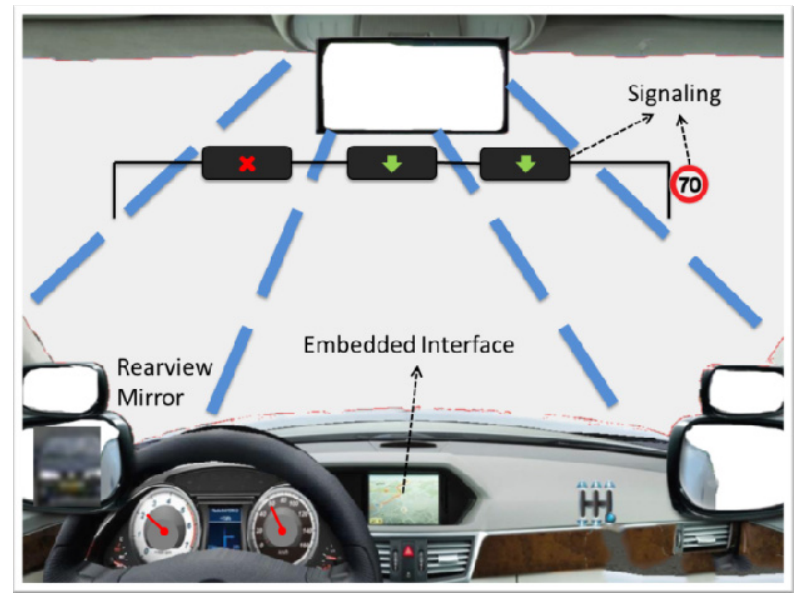

Fig. 5. Interior view of a vehicle in the simulation

Communication and collaboration among the objects in the IoT in the simulation could be: a set of sensors is located on the lane to automatically detect the presence of priority vehicles, and each sensor is in charge of only a certain range of region and is able to notify the management center to perform operations according to their locations (proactive LBS). On the other hand, vehicles with priority could send a priority request to the nearest sensor in front, to initiate lane allocation (reactive LBS). When a priority vehicle leaves the region, the sensor detects it and notifies the management center, which gradually sets the allocation back to normal. It is also the responsibility of a sensor to deliver information about current traffic status within its range of region via displayers such as vertical signaling. The information collected can be disseminated to vehicles via an "on board display". 
Simulator Functionalities. The goal of the simulation is to validate principles and provide support for implementation of the system. Functionalities are described in figure 6.

A scenario editing tool is a complementary tool to the simulator. Its goal is to specify simulation parameters such as lane length, number of lanes, duration of simulation, number of sensors and initial status of lane allocation. This graphic editing tool (Fig. 4) also includes a sensor editor toolkit able to set sensors at appropriate locations in order to adjust the sensor parameters such as range of region in charge, or to test the influence of different placements.

Vehicles, with or without priority, are planned to travel through the area from left to right, with distinctive parameters (speed, initial time, etc.), respecting strictly the signaling of lane allocation throughout the process. A traffic generator tool is able to generate traffic flow, in which there are vehicles with or without priority, and different parameters can be specified, as well as possible itinerary preferences. Moreover, this generator tool helps choose how to launch the traffic flow, with different time intervals and densities.

Simulation visualization can provide opportunities for conducting a usability and acceptability test, which is presented in the following paragraphs.

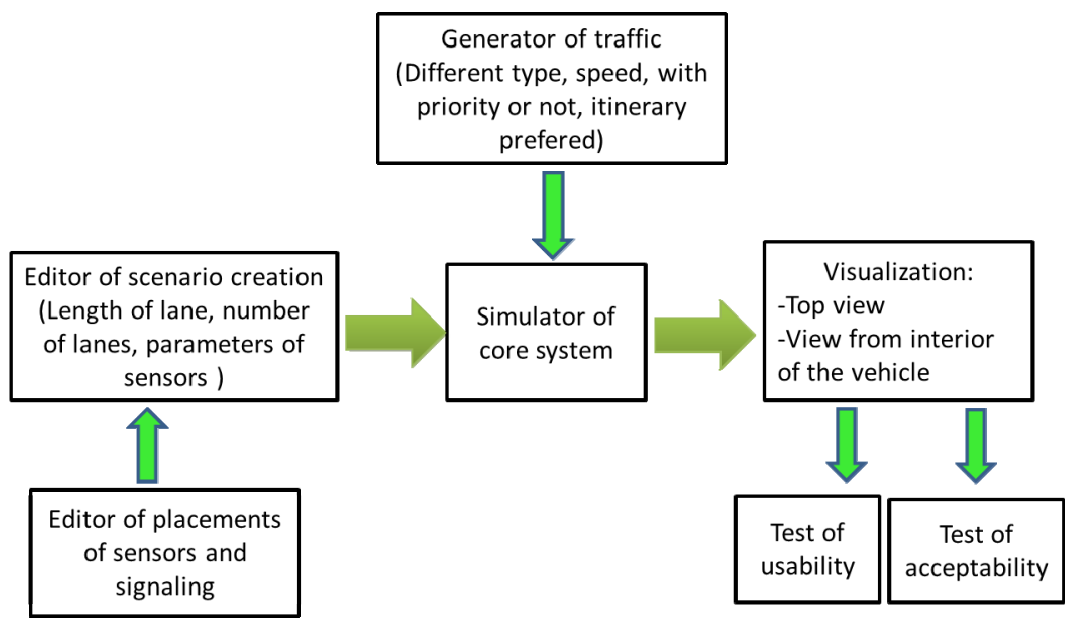

Fig. 6. Simulator functionalities

UI Testing. The simulator goal is to allow ergonomic studies of acceptability of this kind of approach to dynamic lane management. Several levels of tests can be produced, using different views. A map view, as shown in Fig. 4, is used for a global bird's eye view of system functioning. A subjective view, a view from the interior of the vehicle, is used to test users' behaviors. 


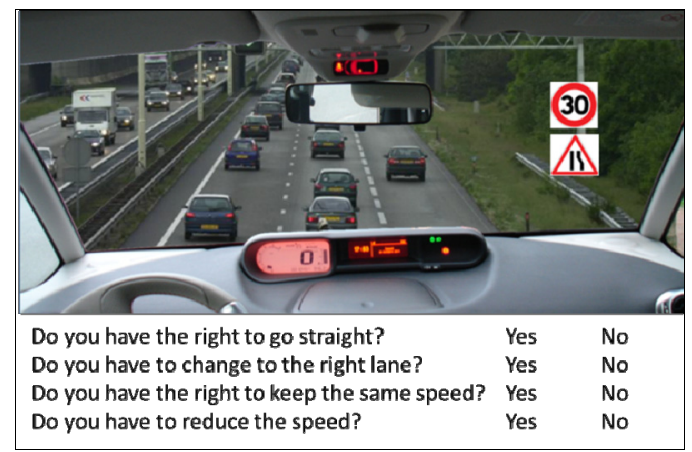

Fig. 7. A scenario of the acceptability study

A scenario screen shot of the acceptability study is shown in Fig. 7. In the test, a set of photos was produced to simulate a static environment. These photos show a multiple lane configuration with insertion of appropriate vertical and horizontal signaling. The pictures are shown to a driver, letting him/ her observe the situation, and then questions are asked, requesting him/ her to choose the appropriate behavior. If the signaling is not easy to remark or understand, the driver may not choose to behave correctly.

The first test study was conducted by our IFSTTAR partners, who studied understandability of appropriate vertical and horizontal traffic signs and road signs. These signs are either static or, in the majority of situations, dynamic (electronic display signs), following dynamic lane evolution. Fig.8 shows several of these signs.
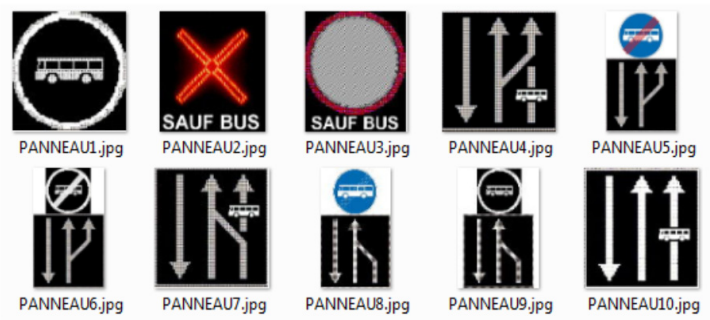

Fig. 8. Traffic signs tested

To increase understanding of acceptability, it is important to study the temporal behavior of drivers. It is not enough to observe appropriate interpretation of driving situations, if the user can take all the time he/ she needs to think about the situation. Rather than creating driving situations by using static photos, our simulation goal is to support dynamic behavior in which the subjective view during simulation is related to driving speed. The situation shown at Fig. 5 can be used to conduct dynamic tests. Speed is not the only dynamic property; external vehicle traffic can also be taken into account, for instance, to what degree a user adapts him/ herself to a more complex situation with the changing of surrounding circumstances such as a car appearing in 
the rearview mirror and the changing of the message on the panel or signaling as well as use of the embedded interface. Examples of the usability test for embedded interfaces are shown in Fig. 9. The GPS, LANE INFOS and the REPORT ACCIDENT one are open to all users, while the PRIORITY page is reserved for active users.
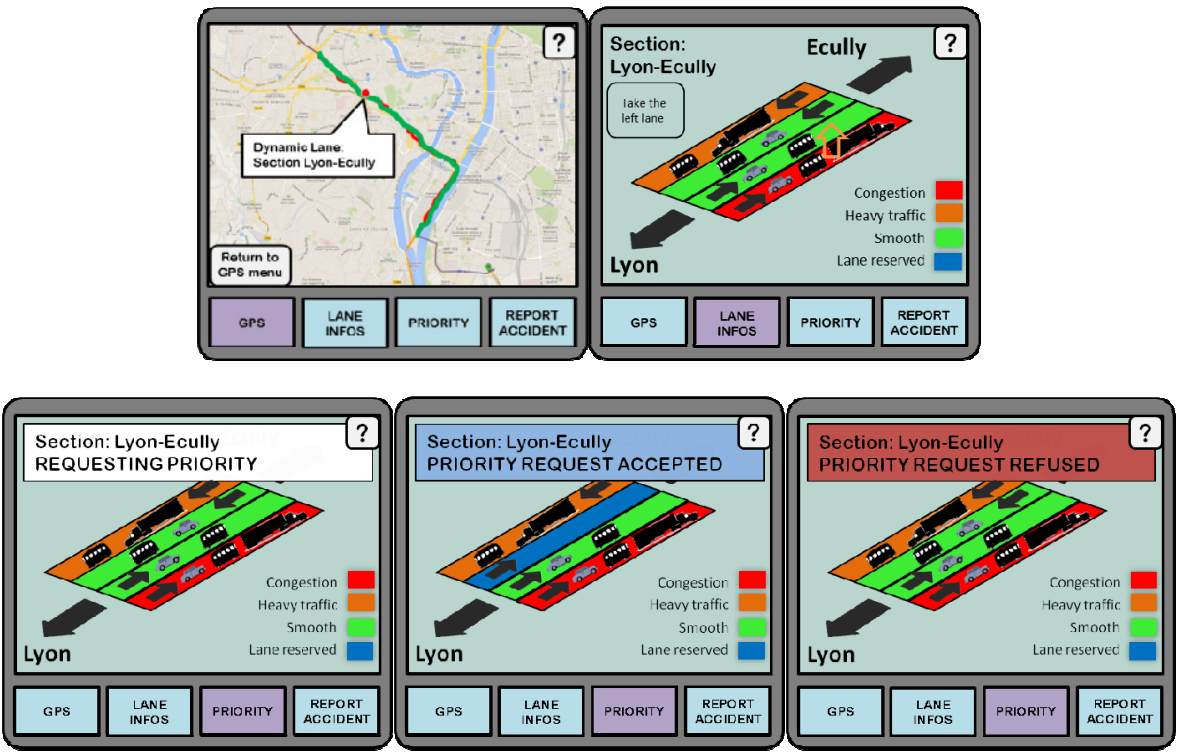

Fig. 9. Usability study of embedded interfaces

\section{Conclusion}

In this paper we briefly explained our view of the Smart City in the context of transportation and citizens' everyday life on which we are currently working $[13,14,15]$. We gave the main principles and techniques used, and presented a precise application based on IoT and LBS. Before in-the-field deployment of this kind of system, it is important to validate it theoretically from technological and human acceptability points of view. For this reason we created a simulator allowing us to design and simulate different scenarios of infrastructure behaviors. We can also connect this simulator to UI testing studies, the goal of which is to validate (or invalidate) acceptability of new driving situations based on the driver's behavior in relation with new vertical and horizontal road signs (Fig. 7). The goal of future work is to increase the realism of our simulator to take into account temporal aspects of driving, allowing us to study the relationship between traffic density and acceptability of dynamicity of lane management. 
Acknowledgements. We extend our thanks to the French Department of Ecology who partially supported this project, and to our colleagues from IFSTTAR, LIRIS, CEA-LETI, EGIS and VOLVO.

\section{References}

1. Xiong, Z.: Smart City and Data Vitalisation. In: The 5th Beihang Centrale Workshop, January 7 (2012)

2. Giusto, D., Iera, A., Morabito, G., Atzori, L.: The Internet of Things. Springer (2010) ISBN: 978-1-4419-1673-0

3. Ambient Intelligence Laboratory, http://www.ami-lab.org, (consulted on April 11, 2013)

4. Weiser, M., Brown, J.: The coming age of calm technology, http: / / www. johnseelybrown.com/calmtech.pdf (consulted on May 11, 2013)

5. Atzori, L., Iera, A., Morabito, G.: The Internet of Things: A survey. Computer Networks 54, 2787-2805 (2010)

6. National Intelligence Council. Disruptive Civil Technologies - Six Technologies with Potential Impacts on US Interests Out to 2025. Conference Report CR 2008-07 (April 2008)

7. Jiang, B., Yao, B.: Location-based services and GIS in perspective. Comput. Environ. Urban Syst. (2006), doi:10.1016/j.compenvurbsys.2006.02.003

8. Bellavista, P., Kupper, A., Helal, S.: Location-Based Services: Back to the Future. IEEE Pervasive Computing 7(2), 85-89 (2008)

9. Csinger, A.: Users models for intent-based authoring. Dessertation. The University of British Columbia (1995)

10. World Geodetic System,

http://en.wikipedia.org/wiki/World_Geodetic_System (consulted on October 12, 2013)

11. Hu, H., Lee, D.: Semantic location modeling for location navigation in mobile environment. In: Proceedings of the 2004 IEEE International Conference on Mobile Data Management, pp. 52-61 (2004)

12. Nouvier, J.: Entrez dans le monde des ITS = Enter the world of ITS, Lyon: CERTU, célérom TU CE12 10450 disponible auprès du département Systèmes du CERTU (2007)

13. David, B., Chalon, R., Favre, B.: ICT and new human-machine interactions for trucks and buses in the future: e-Truck and e-Bus perspectives. In: Kolski, C. (ed.) Human-Computer Interactions in Transport, pp. 157-201. ISTE Ltd and John Wiley \& Sons, Inc. (2011) ISBN: 978-1-84821-279-4

14. David, B., Yin, C., Zhou, Y., Xu, T., Zhang, B., Jin, H., Chalon, R.: SMART-CITY: Problematics, techniques and case studies. In: ICCM 2012, 8th International Conference on Computing Technology and Information Management, Seoul, Korea, pp. 168-174. IEEE Conference Publications (2012) ISBN 978-1-4673-0893-9

15. David, B., Xu, T., Jin, H., Zhou, Y., Chalon, R., Zhang, B., Yin, C., Wang, C.: Useroriented System for Smart City approaches. In: 12th IFAC/IFIP/IFORS/IEA Symposium on Analysis, Design, and Evaluation of Human-Machine Systems, Las Vegas, Nevada, USA, August 11-15, pp. 333-340. IFAC/Elsevier (2013) ISSN 1474-6670. 2013 